\title{
Aneurysm rebleeding before therapy: a predictable disaster?
}

\author{
Marvin Darkwah Oppong, MD, ${ }^{1}$ Meltem Gümüs, ${ }^{1}$ Daniela Pierscianek, MD, ${ }^{1}$ Annika Herten, MD, ${ }^{1}$ \\ Andreas Kneist, MD, ${ }^{1}$ Karsten Wrede, MD, ${ }^{1}$ Lennart Barthel, MD, ${ }^{1}$ Michael Forsting, MD, ${ }^{2}$ \\ Ulrich Sure, MD, ${ }^{1}$ and Ramazan Jabbarli, MD'
}

1Department of Neurosurgery and 2Institute for Diagnostic and Interventional Radiology, University Hospital, University of Duisburg-Essen, Essen, Germany

OBJECTIVE Current guidelines for subarachnoid hemorrhage (SAH) include early aneurysm treatment within 72 hours after ictus. However, aneurysm rebleeding remains a crucial complication of SAH. The aim of this study was to identify independent predictors allowing early stratification of SAH patients for rebleeding risk.

METHODS All patients admitted to the authors' institution with ruptured aneurysms during a 14-year period were eligible for this retrospective study. Demographic and radiographic parameters, aneurysm characteristics, medical history, and medications as well as baseline parameters at admission (blood pressure and laboratory parameters) were evaluated in univariate and multivariate analyses. A novel risk score was created using independent risk factors.

RESULTS Data from 984 cases could be included into the final analysis. Aneurysm rebleeding occurred in 58 cases $(5.9 \%)$, and in 48 of these cases (82.8\%) rerupture occurred within 24 hours after SAH. Of over 30 tested associations, preexisting arterial hypertension ( $p=0.02$; adjusted odds ratio [aOR] 2.56, 1 score point), aneurysm location at the basilar artery ( $p=0.001$, aOR 4.5, 2 score points), sac size $\geq 9 \mathrm{~mm}$ ( $p=0.04$, aOR 1.9, 1 score point), presence of intracerebral hemorrhage ( $p=0.001$, aOR 4.29, 2 score points), and acute hydrocephalus ( $p<0.001$, aOR 6.27, 3 score points) independently predicted aneurysm rebleeding. A score built upon these parameters (0-9 points) showed a good diagnostic accuracy ( $p<0.001$, area under the curve 0.780) for rebleeding prediction.

CONCLUSIONS Certain patient-, aneurysm-, and SAH-specific parameters can reliably predict aneurysm rerupture. A score developed according to these parameters might help to identify individuals that would profit from immediate aneurysm occlusion.

https://thejns.org/doi/abs/10.3171/2018.7.JNS181119

KEYWORDS aneurysm; rebleeding; subarachnoid hemorrhage; vascular disorders

$\mathrm{A}$ NEURYSM rebleeding before therapy is the most relevant preventable cause of death after subarachnoid hemorrhage (SAH) ${ }^{6}$ Furthermore, it is one of the main causes of poor outcome in surviving patients. ${ }^{28}$ Both the European Stroke Organization ${ }^{30}$ and American Stroke Association ${ }^{11}$ have recently issued guidelines recommending aneurysm occlusion in the first 72 hours after ictus (early treatment) as long it is feasible in light of the patient's condition.

Rebleeding rates are still reported to be between $7 \%$ and $21 \% .^{5,33}$ The first 24 hours after initial SAH has been identified as a critical time with the highest rebleeding risk. ${ }^{19,21,22}$ Most of the North American and Western European neurovascular centers have introduced an ultra-early ( $<24$ hours after admission) aneurysm occlusion therapy as standard of care. However, patients who are at risk for early rebleeding might benefit from immediate aneurysm occlusion. Therefore, efforts have been made to identify possible predictors of rebleeding events, but many of these studies have relied on small cohorts and present conflicting results., ${ }^{4,16,18,19,25,26}$ Recent meta-analyses proposed aneurysm size, high systolic blood pressure, high clinical

ABBREVIATIONS aOR = adjusted odds ratio; CSF = cerebrospinal fluid; ICH = intracerebral hemorrhage; IVH = intraventricular hemorrhage; oGS = original Graeb Score; $\mathrm{ROC}=$ receiver operating characteristic; $\mathrm{SAH}=$ subarachnoid hemorrhage; WFNS = World Federation of Neurosurgical Societies.

SUBMITTED April 23, 2018. ACCEPTED July 3, 2018.

INCLUDE WHEN CITING Published online November 30, 2018; DOI: 10.3171/2018.7.JNS181119. 
SAH severity, intraventricular/intracerebral bleeding, and placement of a cerebrospinal fluid (CSF) drainage system as well as aneurysm location as predictors of aneurysm rebleeding. 5,7,31 Additionally, sentinel headache has been reported to be a strong predictor of rebleeding. ${ }^{4}$

The aim of this study was to identify independent risk factors for aneurysm rebleeding in a large institutional SAH cohort. In addition, we aimed to develop a risk score for better estimation of patient-specific rebleeding risk at admission.

\section{Methods}

All consecutive patients treated for aneurysmal SAH in our neurosurgical department between January 2003 and June 2016 were included in this retrospective analysis. This study has been approved by the institutional review board. All persons or their relatives gave their informed consent within a written treatment contract prior to their inclusion in the study.

\section{Treatment Regime}

According to our institutional policy, all SAH patients undergo aneurysm occlusion within 24 hours after admission. Decisions about microsurgical or endovascular treatment were based on interdisciplinary consensus. Acute hydrocephalus (defined as presentation with ventriculomegaly or intraventricular hemorrhage [IVH] and decreased level of consciousness that could not be attributed to other causes than hydrocephalus ${ }^{9}$ ) was treated by drainage of CSF via an external ventricular or lumbar drain. Space-occupying intracerebral hemorrhage (ICH) was treated by urgent surgical decompressive craniectomy and, if applicable, hematoma evacuation. Prior to aneurysm occlusion, a rigorous blood pressure regimen was applied to keep the systolic pressure at $\leq 150 \mathrm{~mm} \mathrm{Hg}$.

An early posttreatment CT scan of the head was performed in the first 24 hours after aneurysm occlusion in all cases. Further imaging was performed as necessary.

All patients were administered oral nimodipine for 21 days and underwent transcranial Doppler ultrasonography daily for 14 days. Symptomatic vasospasm was treated by intraarterial nimodipine application or transluminal catheter angioplasty, if necessary.

\section{Data Management}

Patients' charts were reviewed for demographic and clinical parameters. Imaging was reviewed for all radiographic parameters.

All events involving clinical deterioration before treatment of the aneurysm in conjunction with new hemorrhage on CT were judged as rebleeding events. Rebleeding episodes in referring hospitals, during transport, and at our institution were recorded. Intraprocedural aneurysm rupture or index bleedings following a sentinel headache were not considered rebleeding in this report.

Clinical severity of SAH was assessed according to the World Federation of Neurosurgical Societies (WFNS) grading system. ${ }^{32}$ For statistical analysis, we dichotomized cases into good grade (WFNS grades I-III) and poor grade (WFNS grades IV and V). For assessment of radiographic severity, the original Fisher scale ${ }^{15}$ was used. For analysis, the radiographic severity was dichotomized into low (Fisher grades 1 and 2) and high (Fisher grades 3 and 4) grades.

Patient charts were reviewed for age, sex, preexisting morbidities (arterial hypertension, diabetes mellitus, smoking), and anticoagulation treatment (vitamin $\mathrm{K}$ antagonists, antiplatelet therapy, new oral anticoagulants) prior to admission. Presence of preexisting comorbidities (diabetes mellitus and arterial hypertension) was judged upon the records in the institutional admission protocol, which contains data on past medical history (past illnesses, surgeries, medications, etc.). Furthermore, a wide range of vital and laboratory parameters at admission were also recorded, including blood pressure levels (maximum and minimum values for the systolic and mean overall value for the mean arterial pressure), body temperature (maximum values), and baseline laboratory test values for serum and CSF.

The timings of the bleeding event, hospital admission, and rebleeding event were also documented. Timing of the rebleeding event was stratified into 3 intervals: $<12$ hours, 12-24 hours, and $>24$ hours after the initial bleeding event or admission.

Along with the Fisher grade, the following radiographic parameters were also collected: presence of ICH; presence of IVH; size, location, and shape of the ruptured aneurysm; and the presence of multiple aneurysms. IVH severity was assessed using the original Graeb Score (oGS). ${ }^{17}$ All aneurysms that presented with daughter sac(s) $(<50 \%$ of aneurysm size) or multiple lobes ( $>50 \%$ of aneurysm size) were defined as irregular.

Functional endpoints were in-hospital mortality and poor outcome at 6 months after SAH (defined as modified Rankin Scale ${ }^{35}$ score $>2$ ).

\section{Statistical Analysis and Score Building}

Statistical analysis was performed using SPSS 22 for Mac (IBM Corp.). Continuous variables are presented as mean \pm standard deviation. They were analyzed using the Student t-test for normally distributed and the MannWhitney U-test for nonnormally distributed data. Categorical variables were analyzed using the chi-square test; for samples smaller than 5, the Fisher exact test was used. Statistical significance was set at $p<0.05$. Factors predictive for occurrence of rebleeding in univariate analysis were included in final multivariate binary logistic regression analysis. For continuous variables, a cutoff was identified using the receiver operating characteristic (ROC) curve analysis prior to multivariate analysis. Multivariate analysis was conducted in 2 steps. First, parameters that were present before and those characterizing SAH event were analyzed separately. Then, parameters identified in both analyses were included to the final multivariate model. The results of this cumulative multivariate analysis were used to build a risk score. The adjusted odds ratios (aORs) of these variables were divided by the smallest coefficient and then rounded up to the nearest integer. We used the result to weight the value of each parameter. Finally, the total score value was calculated for each SAH individual in the cohort, depending on the presence of identified risk 


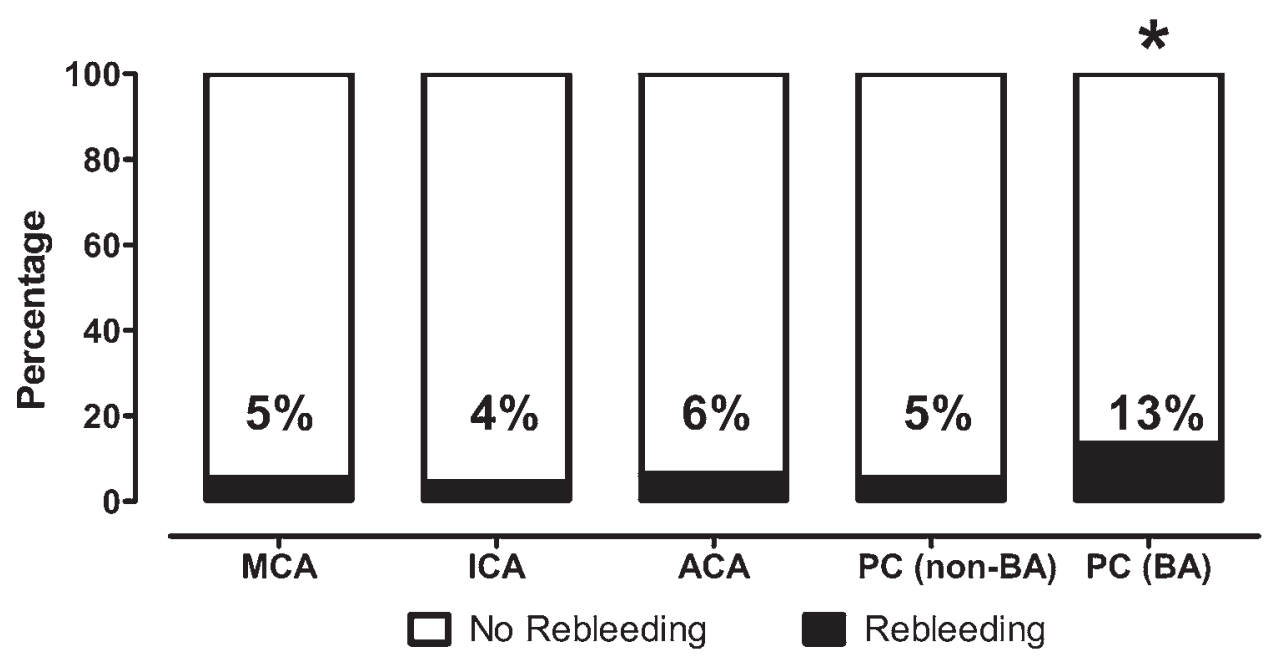

FIG. 1. Rebleeding rate by aneurysm location. The incidence of aneurysms by location for the whole cohort was as follows: anterior cerebral artery (ACA), 374; posterior circulation (PC), 274 (non-basilar artery [BA], 190; BA, 84); middle cerebral artery (MCA), 219; and internal carotid artery (ICA), 117. ${ }^{*} p=0.01$ regarding risk of rebleeding (vs all other locations).

factors for rebleeding. Missing data were replaced using multiple imputations. Diagnostic accuracy of the new score was tested using the ROC curve.

\section{Results}

A total of 994 patients were eligible for this study. Due to initial severe presentation of SAH, a judgment about rebleeding was not possible in 10 cases, and therefore 984 patients were included in final analysis. The majority of the patients were female $(66.9 \%)$ and Caucasian (95.5\%). The mean age at admission was $54.8 \pm 13.6$. Rebleeding occurred in 58 cases $(5.9 \%)$, and in 34 of these cases $(58.6 \%)$ it occurred in the first 12 hours after ictus. In the remaining cases, 14 rebleeding events $(24.1 \%)$ occurred during the next 12 hours post-SAH (i.e., 48 cases within 24 hours). Only 10 (17.2\%) events occurred later in the clinical course. Thirty-two events $(55.2 \%)$ occurred after admission at our institution, 17 (29.3\%) during hospital transport, and $9(15.5 \%)$ at a referring hospital. CSF drainage was required in $674(68.4 \%)$ cases due to acute hydrocephalus. Patients presented in poor initial clinical condition in $41.0 \%$ of cases. Radiographic severity was high in $86.8 \%$ of cases. There was no significant difference in the timing of treatment for the patients with and without rebleeding ( $p=0.28$ for treatment $<8$ hours after admission).

\section{Parameters Present Prior to Admission}

In univariate analysis, higher age at admission (58.9 vs 54.5 years, $\mathrm{p}=0.018$ ), previous use of anticoagulation medication ( $\mathrm{p}=0.02$, OR $2.78,95 \%$ CI $1.22-5.95)$, and premorbid arterial hypertension $(\mathrm{p}=0.01$, OR $2.47,95 \%$ CI 1.21-5.06) were predictive for occurrence of rebleeding. Regarding the characteristics of the ruptured aneurysm, larger size (mean 8.94 vs $7.2 \mathrm{~mm}, \mathrm{p}=0.005$ ) and location at the basilar artery $(p=0.12$, OR $2.74,95 \%$ CI 1.36-5.5; see also Fig. 1) increased the risk of rebleeding significantly. Aneurysm morphology (judged as irregu- lar shape or presence of a daughter sac, $\mathrm{p}=0.88$ and $\mathrm{p}$ $>0.99$, respectively) and presence of multiple aneurysms $(\mathrm{p}=0.32)$ had no predictive value (see also Table 1). For further analysis, aneurysm size was dichotomized as $<9$ $\mathrm{mm}$ versus $\geq 9 \mathrm{~mm}$ and age was dichotomized as $<57$ vs $\geq 57$ years (according to the ROC curves; Supplemental Fig. E1).

In multivariate analysis of parameters prior to $\mathrm{SAH}$, we were able to identify premorbid arterial hypertension ( $\mathrm{p}=$ 0.03 , aOR $2.44,95 \%$ CI 1.10-5.45), aneurysm location at the basilar artery $(\mathrm{p}=0.14$, aOR $2.5,95 \%$ CI $1.2-3.75)$, and sac size $\geq 9 \mathrm{~mm}(\mathrm{p}=0.02$, aOR $2.04,95 \%$ CI $1.11-3.75)$ as independent predictors of aneurysm rebleeding (Table 2).

\section{Clinical and Radiographic Features of SAH}

Patients who initially presented in poor clinical condition had a significantly increased risk of aneurysm rebleeding ( $\mathrm{p}<0.001$, OR 3.45, 95\% CI 1.95-6.11). Higher Fisher grade (grades 3-4, p $=0.006$, OR 8.76, 95\% CI 1.264.02), presence of ICH ( $<<0.001$, OR $2.9,95 \%$ CI $1.7-$ 4.95 ) and higher IVH severity (mean oGS 6.45 vs $4.98, p$ $=0.02$ ), and acute hydrocephalus ( $<<0.001$, OR 6.66, 95\% CI 2.39-18.57; see also Table 1) were also associated with rebleeding risk. The clinically relevant cutoff for IVH severity was set at an oGS of 5 (with an oGS $\geq 5$ considered to indicate severe IVH) (Supplemental Fig. E1).

In multivariate analysis of SAH characteristics, only presence of ICH $(\mathrm{p}=0.004$, aOR $2.42,95 \%$ CI $1.33-4.38)$ and acute hydrocephalus $(\mathrm{p}=0.003, \mathrm{aOR} 5.05,95 \% \mathrm{CI}$ $1.73-14.79)$ independently predicted occurrence of rebleeding (Table 3).

\section{Initial Management of SAH and Laboratory Tests}

Systolic blood pressure $(\mathrm{p}=0.31$ for maximum and $\mathrm{p}=$ 0.71 for minimum), mean arterial pressure $(p=0.89)$, and maximum temperature $(p=0.16)$ at admission did not differ significantly between the 2 groups. A cutoff for maximum systolic blood pressure of $182 \mathrm{~mm} \mathrm{Hg}$ (according to 
TABLE 1. Univariate analysis of parameters predictive for aneurysm rebleeding before treatment

\begin{tabular}{|c|c|c|c|c|}
\hline Parameter & Rebleeding & No Rebleeding & OR (95\% Cl) & p Value \\
\hline No. of patients & $58(5.9 \%)$ & $926(94.1 \%)$ & - & - \\
\hline \multicolumn{5}{|c|}{ Parameters present prior to SAH event } \\
\hline Mean age, yrs & $58.9 \pm 13.58$ & $54.5 \pm 13.68$ & - & 0.02 \\
\hline Sex: female & $65.52 \%$ & $66.95 \%$ & $0.94(0.54-1.64)$ & 0.89 \\
\hline Race: Caucasian & $98.28 \%$ & $95.36 \%$ & $2.78(0.38-20.53)$ & 0.51 \\
\hline Previous anticoagulation & $33.3 \%$ & $15.67 \%$ & $2.69(1.22-5.95)$ & 0.02 \\
\hline Arterial hypertension & $71.05 \%$ & $49.85 \%$ & $2.47(1.21-5.06)$ & 0.01 \\
\hline Smoker (previous or current) & $25.71 \%$ & $23.11 \%$ & $1.15(0.53-2.51)$ & 0.69 \\
\hline Diabetes mellitus & $11.43 \%$ & $5.44 \%$ & $2.24(0.75-6.7)$ & 0.13 \\
\hline Presence of multiple aneurysms & $27.59 \%$ & $34.67 \%$ & $0.72(0.4-1.3)$ & 0.32 \\
\hline Aneurysm location (BA) & $13.1 \%$ & $8.01 \%$ & $2.74(1.36-5.5)$ & 0.01 \\
\hline Mean aneurysm size, $\mathrm{mm}$ & $8.94 \pm 5.07$ & $7.2 \pm 4.64$ & - & 0.005 \\
\hline Irregular shape & $48.98 \%$ & $47.64 \%$ & $1.06(0.59-1.88)$ & 0.88 \\
\hline Daughter sac & $22.45 \%$ & $21.91 \%$ & $1.03(0.52-2.06)$ & $>0.99$ \\
\hline \multicolumn{5}{|c|}{ Clinical \& radiographic features of $\mathrm{SAH}$} \\
\hline WFNS grade IV or V & $68.97 \%$ & $39.2 \%$ & $3.45(1.95-6.11)$ & $<0.001$ \\
\hline Fisher grade 3 or 4 & $98.18 \%$ & $86.04 \%$ & $8.76(1.2-64.02)$ & 0.006 \\
\hline Mean oGS (IVH severity) & $6.45 \pm 3.69$ & $4.98 \pm 3.21$ & - & 0.02 \\
\hline $\mathrm{ICH}$ presence & $53.45 \%$ & $28.39 \%$ & $2.9(1.7-4.95)$ & $<0.001$ \\
\hline Acute hydrocephalus & $93.1 \%$ & $66.95 \%$ & $6.66(2.39-18.57)$ & $<0.001$ \\
\hline \multicolumn{5}{|l|}{ Initial management of $\mathrm{SAH}$} \\
\hline Mean max SBP, mm Hg & $169.8 \pm 27.4$ & $164.8 \pm 26$ & - & 0.31 \\
\hline Max SBP >182 mm Hg & $23.08 \%$ & $17.89 \%$ & $1.38(0.7-2.69)$ & 0.35 \\
\hline Mean min SBP, mm Hg & $121.4 \pm 18.04$ & $123.9 \pm 59.87$ & - & 0.71 \\
\hline Mean arterial pressure, $\mathrm{mm} \mathrm{Hg}$ & $91.11 \pm 14.24$ & $90.69 \pm 14.93$ & - & 0.89 \\
\hline Mean max temperature, ${ }^{\circ} \mathrm{C}$ & $36.99 \pm 0.72$ & $37.07 \pm 1.62$ & - & 0.16 \\
\hline \multicolumn{5}{|c|}{ Lab parameters at admission, mean values } \\
\hline Serum WBC count, $\times 10^{3} / \mu \mathrm{l}$ & $12.64 \pm 4.76$ & $13.1 \pm 4.82$ & - & 0.48 \\
\hline Hemoglobin, g/dl & $12.32 \pm 1.83$ & $12.68 \pm 1.95$ & - & 0.16 \\
\hline CRP in serum, $\mathrm{mg} / \mathrm{dl}$ & $1.5 \pm 3.46$ & $1.15 \pm 2.51$ & - & 0.68 \\
\hline IL-6 in CSF, pg/ml & $1914 \pm 2814$ & $3878 \pm 7043$ & - & 0.37 \\
\hline Cells in CSF, $/ \mu \mathrm{l}$ & $949.2 \pm 1063$ & $1494 \pm 4036$ & - & 0.51 \\
\hline Troponin T in serum, ng/L & $0.345 \pm 1.03$ & $1.78 \pm 25.28$ & - & 0.81 \\
\hline Myoglobin in serum, $\mu \mathrm{l} / \mathrm{L}$ & $79.25 \pm 53.38$ & $134.9 \pm 232.3$ & - & 0.93 \\
\hline $\mathrm{Min} \mathrm{pO}_{2}$ in $\mathrm{BGA}, \mathrm{mm} \mathrm{Hg}$ & $92.23 \pm 21.51$ & $91.4 \pm 39.1$ & - & 0.52 \\
\hline $\mathrm{Max} \mathrm{pCO}_{2}$ in $\mathrm{BGA}, \mathrm{mm} \mathrm{Hg}$ & $40.94 \pm 7.4$ & $39.56 \pm 5.68$ & - & 0.24 \\
\hline
\end{tabular}

$\mathrm{BA}=$ basilar artery; $\mathrm{BGA}=$ blood gas analysis; $\mathrm{CRP}=\mathrm{C}$-reactive protein; IL-6 = interleukin 6; max = maximum; $\min =$ minimum; $\mathrm{SBP}=$ systolic blood pressure; $\mathrm{WBC}=$ white blood cell.

Boldface type indicates statistical significance.

the ROC curves; see Supplemental Fig. E1) also failed to show a significant difference between the 2 groups.

Finally, analysis of the baseline laboratory parameters at admission (serum, CSF, and blood gas tests) did not reveal any valuable laboratory biomarker for aneurysm rebleeding (Table 1).

\section{Construction and Predictive Value of the Risk Score}

All significant risk factors identified in the first-step multivariate analysis could be confirmed as independent rebleeding predictors in final multivariate analysis (Ta- ble 4) and were therefore included in the risk score (0-9 points): premorbid arterial hypertension (1 point, aOR $2.56)$, aneurysm location at basilar artery ( 2 points, aOR 4.5), sac size $\geq 9 \mathrm{~mm}$ (1 point, aOR 1.9), presence of ICH ( 2 points, aOR 4.29 ), and acute hydrocephalus ( 3 points, aOR 6.27; see also Table 5).

The constructed score showed a good diagnostic accuracy for rebleeding prediction $(\mathrm{p}<0.001$, area under the curve 0.78; Supplemental Fig. E2). For patients with a score $<3$, the relative risk of rebleeding was $0 \%$. In $\mathrm{SAH}$ patients with a score $\geq 5$, the relative risk of rebleeding 
TABLE 2. Multivariate analysis of parameters predicting rebleeding that are present before $\mathrm{SAH}$

\begin{tabular}{lccc}
\hline \multicolumn{1}{c}{ Parameter } & aOR & $95 \%$ Cl for aOR & p Value \\
\hline Age $\geq 57$ yrs & 1.44 & $0.78-2.64$ & 0.24 \\
\hline Previous anticoagulation & 1.53 & $0.67-3.50$ & 0.30 \\
\hline Arterial hypertension & 2.44 & $1.10-5.45$ & $\mathbf{0 . 0 3}$ \\
\hline Aneurysm location (BA) & 2.50 & $1.20-5.19$ & $\mathbf{0 . 0 1}$ \\
\hline Sac size $\geq 9 \mathrm{~mm}$ & 2.04 & $1.11-3.75$ & $\mathbf{0 . 0 2}$ \\
\hline
\end{tabular}

Boldface type indicates statistical significance.

was $12.8 \%$. None of the patients reached either of the 2 highest scores of 8 or 9 points. Of the patients with the highest scores within the tested SAH cohort (7 points), $22.2 \%$ developed rebleeding (vs $1.1 \%$ for the remaining SAH cohort, i.e., a 20-fold higher rebleeding risk).

\section{Rebleeding and Outcome}

The rate of in-hospital mortality among patients who suffered rebleeding was 53\%, which was significantly higher than the rate for patients who had no rebleeding event $(15 \%, \mathrm{p}<0.001$, OR $6.45,95 \%$ CI 3.73-11.13; Fig. $2)$. Also the incidence of poor outcome was twice as high among patients with rebleeding (84\% vs $40 \%$, p $<0.001$, OR 7.99, 95\% CI 3.86-16.52; Fig. 3).

\section{Discussion}

The aim of this study was to identify independent risk factors for aneurysm rebleeding prior to therapy. We were able to show that premorbid arterial hypertension, location of the aneurysm at the basilar artery, sac size $\geq 9 \mathrm{~mm}$, presence of $\mathrm{ICH}$, and acute hydrocephalus independently predicted aneurysm rebleeding. As shown in prior publications, rebleeding was strongly associated with poor outcome $4,16,23,28,29$ and mortality. $4,6,16,19,29$

\section{Parameters Present Prior to Admission}

In this study, we evaluated the prognostic value of various potential rebleeding predictors that are known prior to SAH: demographic data, aneurysm characteristics, premorbid conditions, and prior medication. These parameters are of particular interest, since their clinical utility may also apply to patients with unruptured aneurysms. Therefore, these parameters might be used as additional

TABLE 3. Multivariate analysis of parameters predicting rebleeding that are present after initial $\mathrm{SAH}$

\begin{tabular}{lccc}
\hline \multicolumn{1}{c}{ Parameter } & aOR & $95 \%$ Cl for aOR & p Value \\
\hline WFNS grade IV or V & 1.68 & $0.87-3.23$ & 0.12 \\
\hline Fisher grade 3 or 4 & 1.28 & $0.27-6.23$ & 0.75 \\
\hline IVH w/ oGS $\geq 5$ & 1.42 & $0.76-2.65$ & 0.27 \\
\hline ICH & 2.42 & $1.33-4.38$ & $\mathbf{0 . 0 0 4}$ \\
\hline Acute hydrocephalus & 5.05 & $1.73-14.78$ & $\mathbf{0 . 0 0 3}$ \\
\hline
\end{tabular}

Boldface type indicates statistical significance.
TABLE 4. Multivariate analysis of all independent predictors of rebleeding among parameters present prior to and after $\mathrm{SAH}$

\begin{tabular}{lccc}
\hline \multicolumn{1}{c}{ Parameter } & $\mathrm{aOR}$ & $95 \% \mathrm{Cl}$ for aOR & $\mathrm{p}$ Value \\
\hline Arterial hypertension & 2.56 & $1.20-5.48$ & $\mathbf{0 . 0 2}$ \\
\hline Aneurysm location (BA) & 4.50 & $1.91-10.59$ & $\mathbf{0 . 0 0 1}$ \\
\hline Sac size $\geq 9 \mathrm{~mm}$ & 1.90 & $1.03-3.50$ & $\mathbf{0 . 0 4}$ \\
\hline $\mathrm{ICH}$ & 4.29 & $2.26-8.15$ & $<\mathbf{0 . 0 0 1}$ \\
\hline Acute hydrocephalus & 6.27 & $2.22-17.73$ & $\mathbf{0 . 0 0 1}$ \\
\hline
\end{tabular}

Boldface type indicates statistical significance.

arguments in favor of treatment of unruptured aneurysms that are prone to repetitive rupture.

Our findings were similar to those of most previous studies in not showing a correlation between the patients' age $^{4,23,33}$ or $\operatorname{sex}^{4,23,26,33}$ and risk of rebleeding. But there are also conflicting reports stating that male patients have increased rebleeding risk. ${ }^{8,10}$

The size of the ruptured aneurysm is the most discussed risk factor for rebleeding. The majority of authors used cutoffs of $10 \mathrm{~mm} .{ }^{5}$ Despite some conflicting results, ${ }^{19}$ larger aneurysm size is generally accepted as a predictor of aneurysm rebleeding and this status has recently been confirmed by meta-analysis. ${ }^{5,31}$ We identified aneurysm size of $\geq 9 \mathrm{~mm}$ by ROC curve analysis as a cutoff for increased risk of rebleeding. The anterior communicating artery $^{8}$ and the posterior circulation ${ }^{1}$ have been reported as "locations at risk." The authors of a recent meta-analysis reported that aneurysm location in the posterior circulation was predictive for rebleeding, but there were large discrepancies in the pooled data. ${ }^{31}$ Our analysis indicates that specifically the basilar artery, rather than the "posterior circulation" as a whole, is the crucial location increasing rerupture risk. Conflicting with our results, one previous study has found the presence of multiple aneurysms to be predictive for rebleeding. ${ }^{4}$

Regarding aneurysm morphology, there are conflicting results stating that "bump-like" aneurysms are connected to higher rebleeding rates. ${ }^{2,3}$ Other authors state that irregularity ${ }^{1}$ or blebs ${ }^{19}$ are not generally connected to rebleeding events. However, these reports date back to the time before introduction of $3 \mathrm{D}$ angiography $\mathrm{e}^{13,14}$ and consist predominantly of small series. In our series, we could not draw any connection between aneurysm shape and rate of rebleeding events.

The impact of premorbid hypertension on rebleeding events has been the subject of controversy. ${ }^{12,24,33}$ Our find-

TABLE 5. Construction of the risk score

\begin{tabular}{lccc}
\hline \multicolumn{1}{c}{ Parameter } & aOR & Not Weight Adjusted & Weight Adjusted \\
\hline Arterial hypertension & 2.56 & 1.4 & 1 \\
\hline BA aneurysm & 4.50 & 2.4 & 2 \\
\hline Sac size $\geq 9 \mathrm{~mm}$ & 1.90 & 1 & 1 \\
\hline Presence of ICH & 4.29 & 2.3 & 2 \\
\hline Acute hydrocephalus & 6.27 & 3.3 & 3 \\
\hline
\end{tabular}




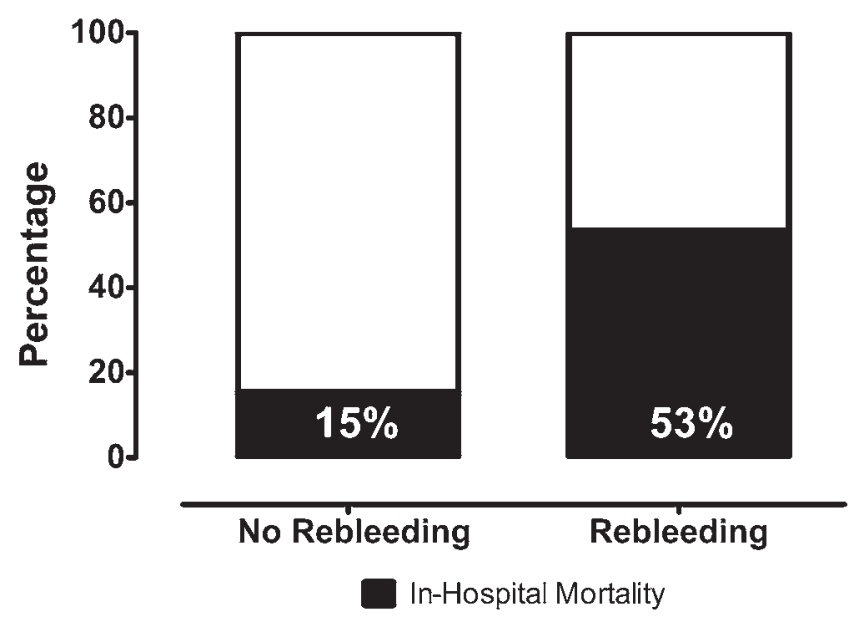

FIG. 2. Risk of in-hospital mortality according to pretreatment rebleeding.

ings are consistent with those of De Marchis et al., ${ }^{12}$ who confirmed premorbid arterial hypertension as an independent risk factor for rebleeding. Smoking also has been reported to increase the risk of rebleeding ${ }^{24}$ but failed to do so in our and other cohorts. ${ }^{4}$

In our cohort, the use of anticoagulants before SAH was significantly associated with rebleeding risk in univariate assessment, but it failed to show independent association in multivariate analysis. Our finding is consistent with results of prior analysis. ${ }^{33}$

\section{Clinical and Radiographic Features of SAH}

Clinical severity of SAH has been identified by the majority of authors to be a risk factor for aneurysm rebleeding, 10,19,22,24,33,34 and therefore it was also included in the risk factors identified in meta-analysis. ${ }^{31}$ There are conflicting results regarding the impact of radiographic severity of SAH on aneurysm rebleeding. ${ }^{4,8,22,33,37}$ Furthermore, IVH and ICH have been reported to predict rebleeding. ${ }^{10,16,}$ ${ }^{26,37}$ In our analysis, only the presence of ICH was independently associated with risk of rebleeding.

Initiation of CSF drainage has been connected with aneurysm rebleeding in previous studies, $7,33,34$ and in our cohort also, patients with acute hydrocephalus had a significantly increased risk of rebleeding.

\section{Initial Management of SAH}

In our series, there was no difference regarding timing of treatment between the rebleeding and the no-rebleeding groups. It has been previously reported that patients with rebleeding events were admitted early after ictus..$^{10,16}$ This might be an effect of the initial higher clinical severity of this group and therefore the more rapid diagnostic workup at external hospitals and/or direct transfer to a neurovascular center. Systolic blood pressure at admission or at transport has been reported to correlate with rebleeding risk. ${ }^{10,16,26,31}$ Like other authors, ${ }^{12,19,22}$ we found that systolic peaks did not increase rebleeding probability in our SAH cohort. This finding may be due to early and rigorous intravenous antihypertensive treatment at our center, limiting such peaks to short intervals without clinical impact.

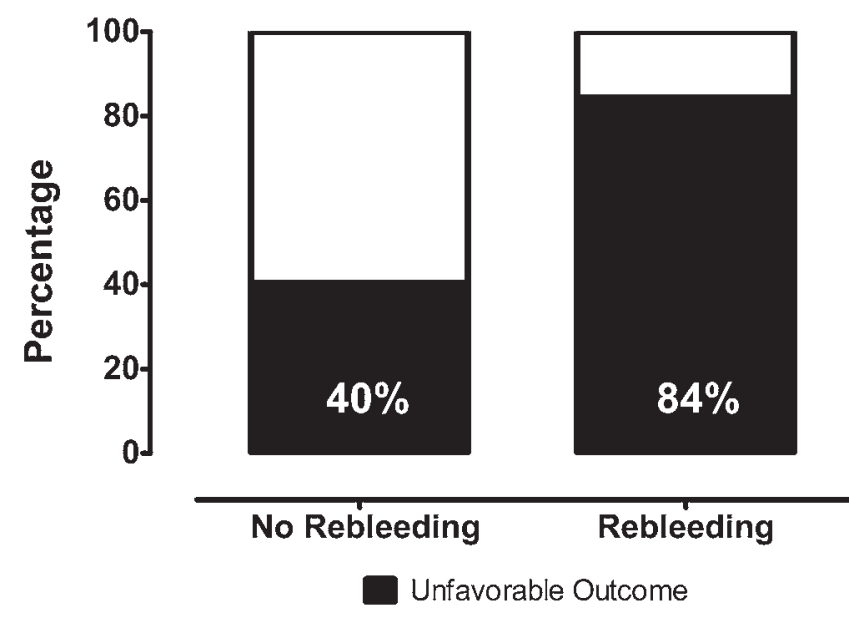

FIG. 3. Risk of poor outcome according to pretreatment rebleeding.

\section{Laboratory Parameters at Admission}

Different parameters have been described as being (thrombin/antithrombin complex, enhancement of platelet sensitivity, ${ }^{16}$ high blood glucose levels, ${ }^{10,22}$ and high white blood cell count ${ }^{10}$ ) or not being (platelet count, ${ }^{10,26}$ PTT [partial thromboplastin time], and bleeding time ${ }^{26}$ ) connected with aneurysm rerupture risk.

None of the laboratory parameters that were tested in our cohort showed any significant correlation with increased rebleeding risk. This included inflammatory parameters in blood and CSF as well as classic cardiac markers. Overall, our data suggest that the role of laboratory parameters might be negligible with respect to the prediction of rebleeding risk.

\section{Implementation of a Score and Clinical Consequences}

Rebleeding was confirmed as a strong contributor to morbidity and mortality in our SAH cohort. Therefore, it is crucial to avoid this preventable occurrence. Many institutions changed from early (first 72 hours) to an ultraearly (first 24 hours) treatment due to the fact that most rebleeding occurs during the first 24 hours after the initial bleeding event. ${ }^{20-22,36}$ It has been suggested that patients would profit from a change in strategy regarding even earlier aneurysm repair. ${ }^{33}$ Retrospective analysis of data from 2 different decades has shown that this change in strategy could lead to a significant reduction in rebleeding rates. ${ }^{27}$ Other authors advocate that a 24-hour immediate aneurysm repair protocol would only affect a very small number of rebleeding events. ${ }^{24}$ Given the fact that a 24-hour emergency treatment protocol might have certain drawbacks, such as uncommon hours of treatment and a need for a higher number of vascular/endovascular neurosurgeons on call, the solution is not necessarily to aim at this standard of care for all patients. One solution might be the early selection and immediate treatment of patients at high risk for rebleeding. In our institution, standard operating procedure for SAH patients includes aneurysm treatment within 24 hours. However, stable patients do not undergo overnight treatment and are instead treated the next day. The proposed score might be used for the triage of tim- 
ing urgency in SAH patients. In particular, patients scoring $\geq 5$ on the risk score should be considered for immediate treatment (regardless of time of day), whereas patients scoring $<3$ can easily be treated according to a standard 24 -hour protocol. Finally, SAH patients with scores $\geq 7$ require special attention from neurosurgeons and neuroradiologists, given the fact that this subgroup accounts for most of the cases with rebleeding in our cohort. Immediate treatment of ruptured aneurysms should be mandatory for patients with these scores.

\section{Limitations}

This retrospective study is burdened with the classic drawbacks of its design, including reduced accuracy and completeness of the recorded data, compared to a prospective cohort. In addition, we were unable to include sentinel headache into our analysis, and it has been reported to be a strong predictor of aneurysm rebleeding before therapy. ${ }^{4}$ Moreover, there was no external validation of the developed score. Nevertheless, we present a detailed evaluation of various factors contributing to aneurysm rebleeding as well as a new risk score for proper estimation of rebleeding risk.

\section{Conclusions}

In this large study, we were able to identify 5 parameters that independently predicted aneurysm rerupture before treatment: premorbid arterial hypertension, location of the aneurysm at the basilar artery, sac size $\geq 9 \mathrm{~mm}$, presence of ICH, and acute hydrocephalus. Furthermore, upon these parameters we developed a score that enables medical staff to determine, using easily accessible information, which patients are at increased risk for rebleeding events. Those patients might profit from rapid treatment with aneurysm occlusion therapy. Further validation of the risk score should be performed.

\section{References}

1. Ando T, Sakai N, Yamada H, Iwai T, Nishimura Y, Hirata $\mathrm{T}$, et al: Analysis of reruptured cerebral aneurysms and the prophylactic effects of barbiturate therapy on the early stage. Neurol Res 11:245-248, 1989

2. Aoyagi N, Hayakawa I: Analysis of 223 ruptured intracranial aneurysms with special reference to rerupture. Surg Neurol 21:445-452, 1984

3. Aoyagi N, Hayakawa I: Study on early re-rupture of intracranial aneurysms. Acta Neurochir (Wien) 138:12-18, 1996

4. Beck J, Raabe A, Szelenyi A, Berkefeld J, Gerlach R, Setzer M, et al: Sentinel headache and the risk of rebleeding after aneurysmal subarachnoid hemorrhage. Stroke 37:2733-2737, 2006

5. Boogaarts HD, van Lieshout JH, van Amerongen MJ, de Vries J, Verbeek AL, Grotenhuis JA, et al: Aneurysm diameter as a risk factor for pretreatment rebleeding: a metaanalysis. J Neurosurg 122:921-928, 2015

6. Broderick JP, Brott TG, Duldner JE, Tomsick T, Leach A: Initial and recurrent bleeding are the major causes of death following subarachnoid hemorrhage. Stroke 25:1342-1347, 1994

7. Cagnazzo F, Gambacciani C, Morganti R, Perrini P: Aneurysm rebleeding after placement of external ventricular drainage: a systematic review and meta-analysis. Acta Neurochir (Wien) 159:695-704, 2017
8. Cha KC, Kim JH, Kang HI, Moon BG, Lee SJ, Kim JS: Aneurysmal rebleeding: factors associated with clinical outcome in the rebleeding patients. J Korean Neurosurg Soc 47:119-123, 2010

9. Claassen J, Bernardini GL, Kreiter K, Bates J, Du YE, Copeland D, et al: Effect of cisternal and ventricular blood on risk of delayed cerebral ischemia after subarachnoid hemorrhage: the Fisher scale revisited. Stroke 32:2012-2020, 2001

10. Cong W, Zhongxin Z, Tiangui L, Zhang Y, Min H, Chao Y: Risk factors for rebleeding of aneurysmal subarachnoid hemorrhage based on the analysis of on-admission information. Turk Neurosurg 22:675-681, 2012

11. Connolly ES Jr, Rabinstein AA, Carhuapoma JR, Derdeyn CP, Dion J, Higashida RT, et al: Guidelines for the management of aneurysmal subarachnoid hemorrhage: a guideline for healthcare professionals from the American Heart Association/American Stroke Association. Stroke 43:1711-1737, 2012

12. De Marchis GM, Lantigua H, Schmidt JM, Lord AS, Velander AJ, Fernandez A, et al: Impact of premorbid hypertension on haemorrhage severity and aneurysm rebleeding risk after subarachnoid haemorrhage. J Neurol Neurosurg Psychiatry 85:56-59, 2014

13. Fahrig R, Fox AJ, Lownie S, Holdsworth DW: Use of a Carm system to generate true three-dimensional computed rotational angiograms: preliminary in vitro and in vivo results. AJNR Am J Neuroradiol 18:1507-1514, 1997

14. Fahrig R, Moreau M, Holdsworth DW: Three-dimensional computed tomographic reconstruction using a C-arm mounted XRII: correction of image intensifier distortion. Med Phys 24:1097-1106, 1997

15. Fisher CM, Kistler JP, Davis JM: Relation of cerebral vasospasm to subarachnoid hemorrhage visualized by computerized tomographic scanning. Neurosurgery 6:1-9, 1980

16. Fujii Y, Takeuchi S, Sasaki O, Minakawa T, Koike T, Tanaka R: Ultra-early rebleeding in spontaneous subarachnoid hemorrhage. J Neurosurg 84:35-42, 1996

17. Graeb DA, Robertson WD, Lapointe JS, Nugent RA, Harrison PB: Computed tomographic diagnosis of intraventricular hemorrhage. Etiology and prognosis. Radiology 143:91-96, 1982

18. Guo LM, Zhou HY, Xu JW, Wang Y, Qiu YM, Jiang JY: Risk factors related to aneurysmal rebleeding. World Neurosurg 76:292-298, 253-254, 2011

19. Inagawa T, Kamiya K, Ogasawara H, Yano T: Rebleeding of ruptured intracranial aneurysms in the acute stage. Surg Neurol 28:93-99, 1987

20. Juvela S: Rebleeding from ruptured intracranial aneurysms. Surg Neurol 32:323-326, 1989

21. Kassell NF, Torner JC: Aneurysmal rebleeding: a preliminary report from the Cooperative Aneurysm Study. Neurosurgery 13:479-481, 1983

22. Kitsuta Y, Suzuki N, Sugiyama M, Yamamoto I: Changes in level of consciousness and association with hyperglycemia as tool for predicting and preventing re-bleeding after spontaneous subarachnoid hemorrhage. Prehosp Disaster Med 21:190-195, 2006

23. Larsen CC, Astrup J: Rebleeding after aneurysmal subarachnoid hemorrhage: a literature review. World Neurosurg 79:307-312, 2013

24. Linzey JR, Williamson C, Rajajee V, Sheehan K, Thompson BG, Pandey AS: Twenty-four-hour emergency intervention versus early intervention in aneurysmal subarachnoid hemorrhage. J Neurosurg 128:1297-1303, 2018

25. Machiel Pleizier C, Algra A, Velthuis BK, Rinkel GJ: Relation between size of aneurysms and risk of rebleeding in patients with subarachnoid haemorrhage. Acta Neurochir (Wien) 148:1277-1280, 2006

26. Ohkuma H, Tsurutani H, Suzuki S: Incidence and signifi- 
cance of early aneurysmal rebleeding before neurosurgical or neurological management. Stroke 32:1176-1180, 2001

27. Park J, Woo H, Kang DH, Kim YS, Kim MY, Shin IH, et al: Formal protocol for emergency treatment of ruptured intracranial aneurysms to reduce in-hospital rebleeding and improve clinical outcomes. J Neurosurg 122:383-391, 2015

28. Roos YB, Beenen LF, Groen RJ, Albrecht KW, Vermeulen M: Timing of surgery in patients with aneurysmal subarachnoid haemorrhage: rebleeding is still the major cause of poor outcome in neurosurgical units that aim at early surgery. $\mathbf{J}$ Neurol Neurosurg Psychiatry 63:490-493, 1997

29. Rosenørn J, Eskesen V, Schmidt K, Rønde F: The risk of rebleeding from ruptured intracranial aneurysms. J Neurosurg 67:329-332, 1987

30. Steiner T, Juvela S, Unterberg A, Jung C, Forsting M, Rinkel G: European Stroke Organization guidelines for the management of intracranial aneurysms and subarachnoid haemorrhage. Cerebrovasc Dis 35:93-112, 2013

31. Tang C, Zhang TS, Zhou LF: Risk factors for rebleeding of aneurysmal subarachnoid hemorrhage: a meta-analysis. PLoS One 9:e99536, 2014

32. Teasdale GM, Drake CG, Hunt W, Kassell N, Sano K, Pertuiset $\mathrm{B}$, et al: A universal subarachnoid hemorrhage scale: report of a committee of the World Federation of Neurosurgical Societies. J Neurol Neurosurg Psychiatry 51:1457, 1988

33. van Donkelaar CE, Bakker NA, Veeger NJ, Uyttenboogaart M, Metzemaekers JD, Luijckx GJ, et al: Predictive factors for rebleeding after aneurysmal subarachnoid hemorrhage: Rebleeding Aneurysmal Subarachnoid Hemorrhage Study. Stroke 46:2100-2106, 2015

34. van Lieshout JH, Pumplün I, Fischer I, Kamp MA, Cornelius JF, Steiger HJ, et al: Volume of cerebrospinal fluid drainage as a predictor for pretreatment aneurysmal rebleeding. $\mathbf{J}$ Neurosurg 128: 1778-1784, 2018

35. van Swieten JC, Koudstaal PJ, Visser MC, Schouten HJ, van Gijn J: Interobserver agreement for the assessment of handicap in stroke patients. Stroke 19:604-607, 1988

36. Yasui N, Suzuki A, Ohta H, Kamiyama H, Kawamura S: [Rebleeding attack of the cerebral aneurysm-clinical significance of the early aneurysmal rebleeding.] No Shinkei Geka 13:61-68, 1985 (Jpn)
37. Zhao B, Fan Y, Xiong Y, Yin R, Zheng K, Li Z, et al: Aneurysm rebleeding after poor-grade aneurysmal subarachnoid hemorrhage: Predictors and impact on clinical outcomes. J Neurol Sci 371:62-66, 2016

\section{Disclosures}

The authors report no conflict of interest concerning the materials or methods used in this study or the findings specified in this paper.

\section{Author Contributions}

Conception and design: Darkwah Oppong. Acquisition of data: Darkwah Oppong, Gümüs, Jabbarli. Analysis and interpretation of data: Darkwah Oppong, Jabbarli. Drafting the article:

Darkwah Oppong, Jabbarli. Critically revising the article: Gümüs, Pierscianek, Herten, Kneist, Wrede, Barthel, Forsting, Sure, Jabbarli. Reviewed submitted version of manuscript: Sure, Jabbarli. Approved the final version of the manuscript on behalf of all authors: Darkwah Oppong. Statistical analysis: Darkwah Oppong, Jabbarli. Administrative/technical/material support: Sure. Study supervision: Jabbarli.

\section{Supplemental Information}

\section{Online-Only Content}

Supplemental material is available with the online version of the article.

Supplemental Figs. E1 and E2. https://thejns.org/doi/suppl/ 10.3171/2018.7.JNS181119.

\section{Correspondence}

Marvin Darkwah Oppong: University Hospital Essen, Germany. marvin.darkwahoppong@uk-essen.de. 